\section{References}

1. Schwartz JT, Brotman AW. Drugs 44: 981-992, 1992

2. Cole J. Maclean Hosp J 7: 61-87, 1992

3. Farmer AE, Blewett A. Drugs 45: 374-383, 1993

4. Bristow MF, Hirsch SR. Drug Safety 8: 136-148, 1993

5. Marder SR, et al. Arch Gen Psychiatry 44: 518-521, 1987
6. Kane J. J Clin Psychiatry 45: 5-12, 1984

7. Cohen LS, et al. Psychosomatics 30: 25-33, 1989

8. Rosenberg MR, Green M. Arch Intern Med 149: 1927-1931, 1989

9. Adler LA, et al. Psychopharmcol 97: 1-11, 1989

10. Kane JM. Br J Psychiatry 160 (Suppl. 17): 41-45, 1992

11. Kaplan HI, Saddock BJ. In Comprehensive textbook of psychiatry, p. 1511 , Williams \& Wilkins, Baltimore, 1985

12. Snyder SH. Am J Psychiatry 138: 460-464, 198

\title{
Regional Considerations in the Use of Antipsychotic Drugs
}

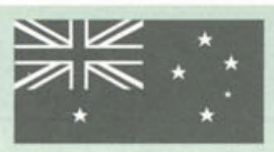

\section{In Australia}

Newly-diagnosed patients with recentonset psychosis are drug-naïve, so the drug to which they will best respond, and the pattern of adverse effects that will occur, are unknown.

Commencing with a very low dosage of haloperidol or pimozide ( $2 \mathrm{mg} /$ day) and building up to a dosage of 5 to $10 \mathrm{mg} /$ day, adverse effects permitting, then assessing the response after 2 to 3 weeks is most appropriate. Behavioural disturbances in the acute phase should be managed with supportive nursing care and liberal short term use of benzodiazepines. Droperidol may be more appropriate than haloperidol in patients who are acutely agitated.

It may be possible to reserve the use of depot antipsychotics for exceptional cases, as depot medication is not necessarily the mainstay of long term therapy.

There is evidence that much lower dosages of antipsychotics are effective, both in the acute and maintenance settings. This should greatly assist compliance.

Apart from antipsychotics, the sequence of drugs to be utilised in treatment-resistant patients remains unclear. However, lithium and ECT may have a more important role in refractory acute schizophrenia.

Patrick D. McGorry, Parkville, Vic.

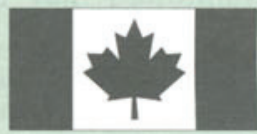

\section{In Canada}

In Canada, high-potency antipsychotics such as haloperidol are preferred. They cause fewer adverse effects than low-potency antipsychotics, except for extrapyramidal problems, which can be controlled by prophylactic anti-Parkinsonian medication. Haloperidol, combined with a benzodiazepine, is the preferred treatment for a very agitated patient.

High-potency antipsychotics are effective in $\leq 75 \%$ of patients with acute schizophrenia. However, after 2 to 12 years of follow up, only $20 \%$ of young, chronically schizophrenic patients have a good outcome. In addition, the risk of tardive dyskinesia increases to $40 \%$ in patients $>40$ years old during long term administration of typical antipsychotics. After discharge (especially in newly-diagnosed patients), depot antipsychotics are indicated, as patients have little insight into their illness.

This may change with the availability of risperidone (see Drugs \& Therapy Perspectives 1: 7, 5 Jul 1993 - Ed.), which is superior to haloperidol in treating chronic schizophrenia. It also effectively treats acutely ill patients, but does not appear to be more effective than haloperidol in this patient group.

Risperidone has a similar therapeutic profile to clozapine in chronic treatment-resistant schizophrenia. It is superior to typical antipsychotics, particularly with regard to negative symptoms, and produces fewer extrapyramidal symptoms, without significant haematological effects.

\section{In USA}

Many antipsychotics used elsewhere are not licensed in the US, including benzamides and newer atypical drugs, as well as depot agents other than esters of fluphenazine or haloperidol.

For acute psychosis and mania, moderate antipsychotic dosages are commonly combined with sedating dosages of a potent, injectable benzodiazepine (e.g. lorazepam). This reduces the risk of adverse effects. For affective psychoses, an antipsychotic is usually combined with an antidepressant or mood-stabilising agent, as monotherapy is less effective in such cases. Complex regimens are appearing for treatment-resistant chronic affective psychoses. They include mixtures of antipsychotic, mood-altering, sedative and anticonvulsant agents.

Clozapine has gained acceptance despite concern over serious adverse effects (seizures, weight gain, tachycardia and fever are much more common than leucopenia) and the indefinite requirement for white blood cell counts. It is sometimes combined with valproic acid and is increasingly used in patients with otherwise unresponsive affective psychotic disorders.

In geriatric patients, low dosages are standard and low potency agents are avoided. Antipsychotics of intermediate potency (e.g. perphenazine) may be preferred to minimise the risk of Parkinsonism or autonomic and central confusion that may occur with highand low-potency agents, respectively.

Ross J. Baldessarini, Belmont, MA

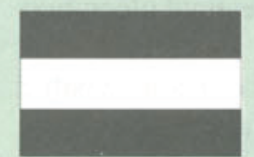

\section{In Austria}

In general, the practice of antipsychotic use in Austria corresponds well with the recommendations of the article, although the choice of drug may differ. Austrian psychiatrists frequently use sulpiride. Clopenthixol is also commonly prescribed, often in the depot formulation that is available on the Austrian market.

Combining antipsychotics is still common practice in Austria, but this may change once the guidelines and recommendations from clinical trial results filter their way through into routine practice. Austrian psychiatrists also still quite frequently use intravenous antipsychotics in acutely-ill schizophrenic patients.

It is important to check plasma antipsychotic concentrations before diagnosing a patient as nonresponsive. Clozapine is usually considered earlier in the course of therapy for treatment-resistant patients, certainly before ECT.

For maintenance treatment, Austrian psychiatrists use depot preparations in about $50 \%$ of patients, which is considerably more than in the US, for example. In contrast, antipsychotic treatment is only continued for relatively short periods (especially after the first episode), which does not comply with recent guidelines [in Kissling W (Ed.), Guidelines for neuroleptic relapse prevention in schizophrenia, pp. 155-163, Springer Verlag, Berlin, 1991]. 